\title{
Fluoroscopically Guided Caudal Epidural Steroid InJections for Lumbar Spinal Stenosis: A Restrospective Evaluation of Long TERM EFFICACY
}

\author{
Lisha Barré, MD, Gregory E Lutz, MD, Daniel Southern, MD, and Grant Cooper, MD
}

\begin{abstract}
Background: Degenerative lumbar spinal stenosis is a frequent cause of disability in the elderly population. Epidural steroid injections are a commonly used conservative modality in the treatment of patients with degenerative lumbar spinal stenosis. Relatively few studies have specifically addressed the efficacy of epidural steroid injections for spinal stenosis, with success rates varying from $20 \%$ to $100 \%$.

Objective: To assess the efficacy of fluoroscopically guided caudal epidural steroid injections in the management of degenerative lumbar spinal stenosis.

Design: Retrospective chart review and follow-up study.

Methods: All the patients who had undergone at least one fluoroscopically guided caudal epidural steroid injection between
\end{abstract}

Degenerative lumbar spinal stenosis (DLSS) commonly results in lower extremity radicular and low back pain (LBP). It is a frequent cause of disability in the elderly population. The symptoms result from bony and soft tissue changes that lead to narrowing of the spinal canal causing mechanical compression of the lumbar spinal nerve roots. Symptomatic DLSS is usually due to some combination of central, lateral recess, and foraminal stenosis. Ligamentum flavum thickening, zygapophyseal joint hypertrophy, disk bulges, and degenerative spondylolisthesis are the most common contributors to stenotic narrowing $(1,2)$.

The diagnosis of spinal stenosis is being made with increasing frequency using magnetic resonance imaging or CT with

From Physiatry Department, Hospital for Special Surgery, New York, NY.. Address Correspondence: Gregory E. Lutz, MD, Physiatrist-in-chief, Hospital for Special Surgery, 535 East 7oth Street, New York, NY 10021.

E-mail: lutzg@hss.edu

Support: Nothing of value received from a commercial entity related to this research

Conflict of Interest: None
1995 and 2002 were reviewed.

All of the caudal epidural steroid injections were done with fluoroscopic guidance.

Main Outcome Measures: Visual Numeric Scale (VNS), Roland-Morris Disability Questionnaire (RMDQ), North American Spine Society Patient Satisfaction Index (NASS), and subsequent surgery.

Results: Ninety-five patients selected from chart review met inclusion criteria. Eighty (84\%) completed the follow-up questionnaire by mail or telephone Interview. Patients received an average of 1.6 epidural steroid injections. Twelve patients subsequently underwent surgical procedures. A VNS improvement of $50 \%$ or greater was seen in $35 \%$ of patients. A functional improvement of 2 points or greater was seen on the RMDQ in
$36 \%$ of patients. Long-term success of treatment was seen in $35 \%$ of patients. The concurrent presence of degenerative spondylolisthesis was the only variable which was found to have a significant positive correlation with successful outcomes $(P<0.009)$.

Conclusion: Caudally placed fluoroscopically guided epidural steroid injections offered a safe, minimally invasive option for managing pain caused by lumbar spinal stenosis. The concurrent presence of degenerative spondylolisthesis appears to be and independent positive prognostic factor for successful outcome.

Keywords: Epidural steroid injection, spinal stenosis, fluoroscopy

myelography (3). However, severity of radiographic stenosis has been shown to correlate poorly with symptom severity (4-6). Treatment options currently available include both conservative and surgical approaches.

DLSS is the most common reason for performing lumbar spinal surgery in adults over the age of $65(7,8)$. Decompressive treatment usually consists of lumbar laminectomy with or without fusion. Reported successful outcomes range from $57-91 \%(9-14)$.

Numerous studies have evaluated the efficacy of non-surgical treatments or compared conservative and surgical outcomes $(11,13-18)$. These studies report widely varying success rates (19). At least partly underlying this variability is the fact that these studies frequently fail to delineate a specific conservative therapy. Multiple modalities, including therapeutic exercise, analgesics, ergonomic training, and epidural steroid injections are grouped under the label conservative therapy. Without distinguishing between the various approaches it is difficult to clearly assess efficacies.
Relatively few studies have specifically addressed the efficacy of epidural injections for spinal stenosis (20-24), more than 40 publications have described results with epidural injections for low back pain and radiculopathy $(25,26)$. Reported success rates are highly variable, ranging from $20 \%-100 \%$ (27).

Several factors can be implicated in this tremendously wide ranging variability. First, previous reports have non-selectively evaluated a mixture of epidural delivery routes including caudal, transforaminal, and interlaminar, each of which may have different efficacies. Second, many previous reports have failed to distinguish between different etiologies of back and leg pain, so that patients with disc herniations, spondylolisthesis, stenosis, facet syndromes and other pathologies are non-selectively included in the same study. Further, distinctions between symptomatology, such as axial low back pain verses radicular low back pain, have rarely been made (28). Finally, previous reports often have failed to use fluoroscopic guidance with pre-injection contrast used to document proper flow to 
the target level. Without this documentation, the epidural injectate may be misplaced in up to $30 \%$ of transforaminal and $40 \%$ of caudal cases, even in skilled hands (29-33).

No study to date has specifically addressed the efficacy of fluoroscopic caudal epidural steroid injections as a treatment for patients with radiographic and clinical evidence of symptomatic lumbar spinal stenosis. The objective of our current study, therefore, was to retrospectively evaluate the efficacy of fluoroscopicallyguided caudal epidural steroid injections in this specific patient population.

\section{Methods}

We retrospectively reviewed the charts of patients with lumbar spinal stenosis who had undergone at least one fluoroscopically guided caudal epidural steroid injection between 1995 and 2002 at the academic-affiliated outpatient physiatry practice of the senior author (G.E.L.). All patients included in the study had documented magnetic resonance imaging (MRI) findings of lumbar central spinal stenosis, lateral stenosis or a combined stenotic picture. Central stenosis was defined as overall narrowing of the anteroposterior diameter of the spinal canal secondary to ligamentum flavum hypertrophy, central disk protrusion, zygapophyseal joint hypertrophy and/or degenerative spondylolisthesis . Lateral stenosis, including lateral recess and foraminal stenosis, was limited to those cases resulting from zygapophyseal joint hypertrophy or other bony pathology; we did not include cases with lateral disk herniations. This exclusion was made because our goal was to examine stenosis exclusively.

Patients were eligible for study inclusion if they had degenerative lumbar spinal stenosis resulting in back or bilateral leg pain for 3 months or greater; if they had failed conservative treatment including physical therapy and NSAIDS; and if they had undergone at least one caudally placed fluoroscopically guided epidural steroid injection. Patients were excluded if they had undergone previous lumbar spinal surgery.

All of the caudal epidural steroid injections were done with fluoroscopic guidance. The patients were placed prone on the fluoroscopy table and prepped and draped in a sterile fashion. Following local anesthesia, a 22 gauge, 2.5 inch spinal nee- dle was advanced through the sacrococcygeal ligament into midline position in the sacral epidural space. Anteroposterior and lateral radiographs confirmed proper needle placement. Iohexol (Omnipaque 180)

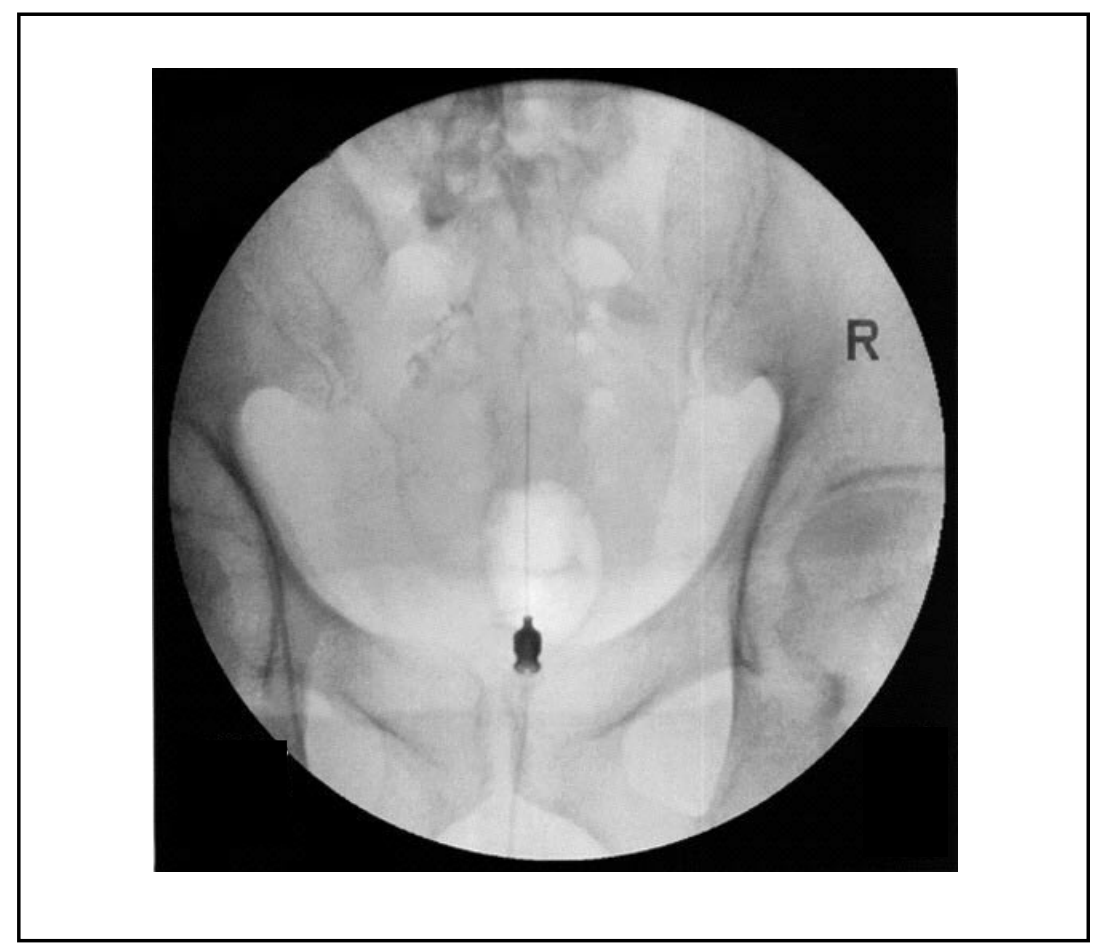

Fig. 1. Fluoroscopic AP view of caudally placed epidural steroid injection

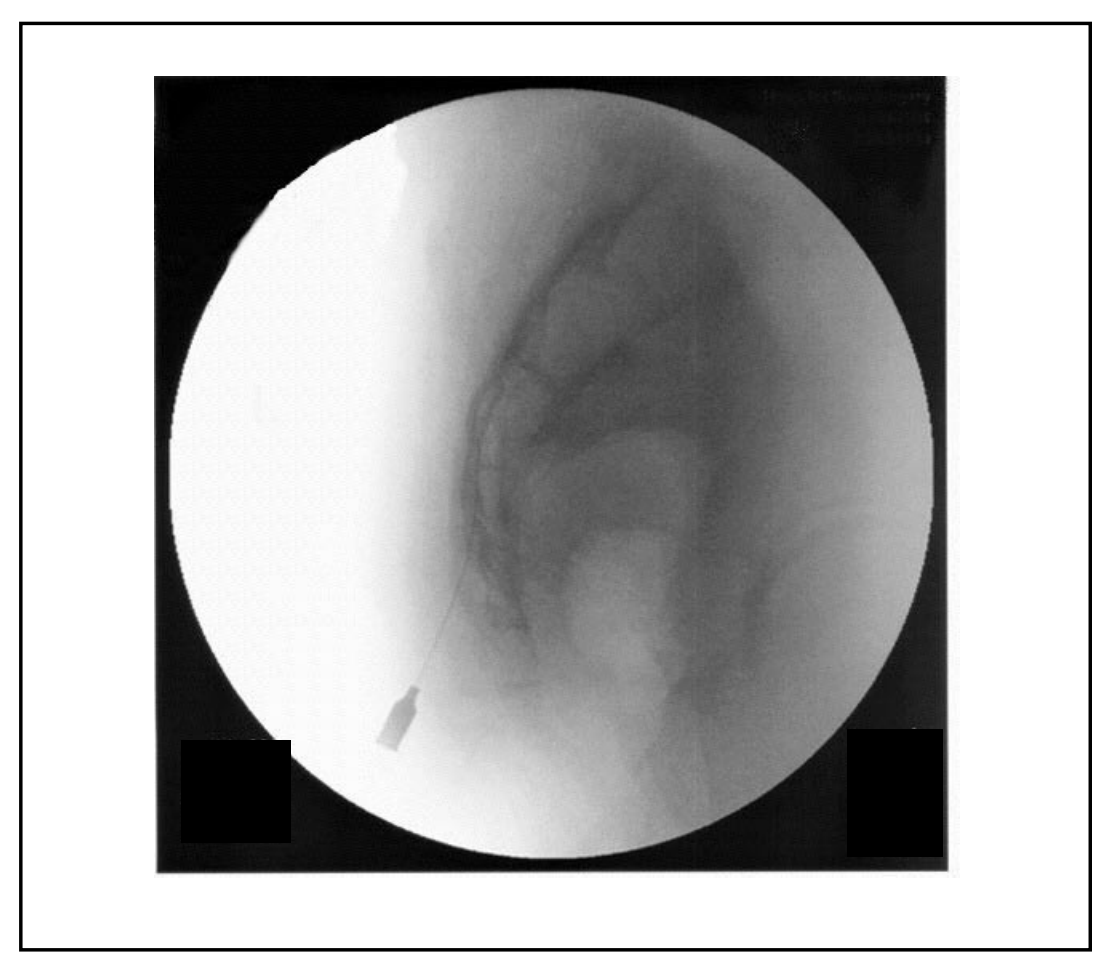

Fig. 2. Fluoroscopic lateral view of caudally placed epidural steroid injection 
Table 1. Patient Satisfaction Index (North American Spine Society)

\begin{tabular}{|c|l|}
\hline Score & \\
\hline $\mathbf{1}$ & Epidural steroids met my expectations \\
\hline $\mathbf{2}$ & $\begin{array}{l}\text { I improved less than I had hoped, but I would undergo the same procedure again } \\
\text { for the same result }\end{array}$ \\
\hline $\mathbf{3}$ & $\begin{array}{l}\text { Epidural steroids helped, but I would not undergo the same procedure again for } \\
\text { the same result }\end{array}$ \\
\hline $\mathbf{4}$ & I am the same or worse than before epidural steroids \\
\hline
\end{tabular}

Table 2. Outcome criteria following caudal epidural steroid injections

\begin{tabular}{|c|c|c|c|c|}
\hline & $\begin{array}{c}\text { Post-Epidural } \\
\text { Surgery }\end{array}$ & $\begin{array}{c}\text { NASS } \\
\text { Satisfaction }\end{array}$ & $\begin{array}{c}\text { VNS } \\
\text { Improvement }\end{array}$ & $\begin{array}{c}\text { Roland-Morris } \\
\text { Improvement }\end{array}$ \\
\hline Success & No & $1-2$ & $\geq 2$ points & $\geq 2$ pts \\
\hline Failure & Yes & $3-4$ & $<2$ pts & $<2$ pts \\
\hline
\end{tabular}

al spread was documented, $80 \mathrm{mg}$ of triamcinolone and $8 \mathrm{cc}$ of $0.5 \%$ preservative free lidocaine were injected. The needle was then removed and patients were monitored for any adverse reactions. All injections were performed by the principle investigator (GEL).

Patients were followed at 1 week. If they reported significant relief, no further injections were scheduled at that time. If, however they reported partial pain relief, a second epidural was scheduled two weeks following the first injection. Up to three injections were scheduled at successive two-week intervals. For those patients who received no pain relief after the first injection no further injections were performed. All patients undergoing caudal epidural steroid injections were concurrently enrolled in a 4 to 6 week physical therapy program for flexion based lumbar stabilization and stretching exercises.

Information collected from the retrospective chart review included sex, age at presentation, level or levels of spinal stenosis, the presence of concurrent spondylolisthesis, the level of spondylolisthesis if present, the distribution of pain, the duration of symptoms at presentation, the number and dates of all fluoroscopic caudal epidural steroid injections, and the patient's pain score on the 10 point Verbal Numeric Pain Scale (VNS) at initial clinical presentation. Independent investigators performed all chart reviews.

Patients who met inclusion criteria were sent follow-up questionnaires. The questionnaires consisted of the following: (1) 10 point VNS scale asking the patient to recall his or her worst pain level prior to the caudal epidural steroid injection; (2) 10 point VNS assessing the worst pain level during the previous week; (3) A ques- tion regarding whether back surgery or other procedures had occurred since the procedure; (4) the 4 point North American Spine Society (NASS) Patient Satisfaction Index (Table 1); and (5) the 24 item Roland-Morris Disability Questionnaire (RMDQ), which requested disabili-

ty assessment both prior to and following the injection. Personnel who were not involved in treatment carried out telephone interviews of those patients who failed to return the questionnaires. Likewise, independent investigators undertook review of the returned questionnaires.

To assess patient response to caudal epidural steroid injections we compared pre-injection VNS and RMDQ scores as unavailable, as recalled by the patient (i.e., as reported on the follow-up questionnaires or telephone interviews) to postinjection scores. A clinically successful outcome was defined as a VNS improveinitially recorded in the charts or, when

Table 3. Demographic and clinical attributes

Table 4. Clinical features ment of 2 points or greater, a positive NASS satisfaction score (indicating patient satisfaction with the final outcome), and a RMDQ improvement of 2 points or greater. Those patients who failed to meet these criteria or who, subsequent to undergoing caudal epidural steroid injections, underwent lumbar surgical procedures were considered to have failed treatment (Table 2).

Statistical analysis of the data were performed using SPSS Version 9.0. The demographic and clinical attributes of successes verses failures were evaluated using Pearson Chi-Square or Fisher's Exact test for nominal data. For non-parametric ordinal data Mann-Whitney test was used. Parametric data analysis was performed using the independent samples t-test. The alpha level was set at 0.05 .

\section{REsUlts}

A total of 4,756 charts were reviewed and 97 patients met inclusion criteria. Of these, two were deceased, leaving 95 patients for evaluation, $80(84 \%)$ of whom completed the follow-up questionnaire either by mail or telephone interview. Forty-five women and 35 men comprised this group. The average patient age at presentation was 69 years (range: 40-91 years) and the mean duration of symptoms prior to the procedure was 41 months (range 3-360 months). The mean follow-up time was 32 months (range: 5-76 months) (Table 3).

Patients received an average of 1.6 caudal epidural steroid injections. Thirty-three patients (41\%) had degenerative spondylolisthesis by MRI. Fifty-one

\begin{tabular}{|c|c|c|c|}
\hline & Mean & Standard Deviation & Range \\
\hline Age & $69 \mathrm{yrs}$ & $9.8 \mathrm{yrs}$ & $40-91 \mathrm{yrs}$ \\
\hline Symptom Duration & $41 \mathrm{mo}$ & $69 \mathrm{mo}$ & $3-360$ \\
\hline Follow-up & $32 \mathrm{mo}$ & $19 \mathrm{mo}$ & $5-76 \mathrm{mo}$ \\
\hline Number of Injections & 1.6 & 0.88 & $1-6$ \\
\hline
\end{tabular}

\begin{tabular}{|l|c|c|}
\hline & Patients & Percent \\
\hline Spondylolisthesis & 33 & $41 \%$ \\
\hline Single level stenosis (L4-5 in 78\%) & 51 & $64 \%$ \\
\hline Multiple level stenosis & 29 & $36 \%$ \\
\hline Axial pain only & 18 & $23 \%$ \\
\hline Leg pain below knee & 47 & $58 \%$ \\
\hline Leg pain above knee & 15 & $19 \%$ \\
\hline
\end{tabular}


Table 5. Outcomes

\begin{tabular}{|l|c|c|}
\hline & Patient number & Percent \\
\hline Surgical procedure & 12 & $15 \%$ \\
\hline VNS change $\geq 2$ & 40 & $50 \%$ \\
\hline RMDQ change $\geq \mathbf{2}$ & 29 & $36 \%$ \\
\hline Positive NASS satisfaction score & 34 & $42 \%$ \\
\hline Overall success & 28 & $35 \%$ \\
\hline
\end{tabular}

Table 6. Outcomes in Spondylolisthesis v. Non-Spondylolisthesis Patients

\begin{tabular}{|l|c|c|c|}
\hline & Success & Failure & Total \\
\hline Spondylolisthesis present & 17 & 16 & $33(51.5 \%)$ \\
\hline Spondylolisthesis absent & 11 & 36 & $47(30.5 \%)$ \\
\hline Total & 28 & 52 & $80(35.5 \%)$ \\
\hline
\end{tabular}

A positive correlation was found between the presence of spondylolisthesis and a successful outcome following caudal ESI $(P=$. 009)

patients $(64 \%)$ had single level stenosis, while 29 (36\%) had multiple level pathology. The most common level of stenosis was L4-5, encompassing $78 \%$ of the single level stenotics. Regarding pain radiation patterns, 18 patients $(23 \%)$ presented with axial back pain only, while the remainder had bilateral leg symptoms. Of those with leg pain, $75 \%$ reported radiation of pain below the level of the knee; only $25 \%$ had radiation that did not extend beyond the knee (Table 4).

The average recalled VNS and RMDQ pre-injection scores were 7.9 and 12.1 respectively. Forty patients (50\%) acknowledged a VNS improvement of 2 points or more at follow-up, with 28 $(35 \%)$ of these patients having at least a $50 \%$ improvement. An RMDQ improvement of 2 points or greater was reported by 29 patients $(36 \%)$.

With regard to patient satisfaction as measured by the NASS index, 34 patients $(42 \%)$ reported that the procedure had fully met their expectations or that they would undergo the procedure again for the same outcome.

Twelve patients required surgical treatment. The mean age of patients in the surgery group was significantly younger at $63(\mathrm{P}=0.002)$. Otherwise there were no statistically significant differences between the surgical and nonsurgical patients.

The overall long-term success rate, as defined in the methods section, was 35\% (28 patients) (Table 5). However, when responders were compared to non-responders, a strong positive correlation was found between the presence of spondylolisthesis and a successful outcome fol- lowing caudal epidural steroid injections $(P=0.009)$ (Table 6). Otherwise, there were no significant differences between responders and nonresponders in terms of age, sex, pain distribution patterns, duration of symptoms, follow-up time, extent of stenosis (i.e. multiple verses single level), total number of injections, baseline VNS scores (whether recalled or recorded at initial presentation) or baseline RMDQ scores.

An evaluation of patients with spondylolisthesis verses the non-spondylolisthesis patients revealed that the former were significantly older, with an average age of $73(\mathrm{P}=0.003)$, and had a higher frequency of single versus multiple level stenosis.

There were no reported major complications such as infection, dural tear, or nerve injury following any of the procedures.

\section{DISCUSSION}

Robecchi and Capra (34) first reported the use of epidural steroid injections for the alleviation of sciatic pain in 1951 in Europe. In the United States, Benzon (35) reported that epidural steroid injections were first used for radicular pain in 1960. Since that time over a hundred papers have been published on the use of epidural steroid injections for the treatment of low back pain of various etiologies (36). Studies that specifically assess the use of epidural steroid injections in the treatment of DLSS are relatively sparse. Cuckler et al (24) conducted a prospective, randomized, double blind study of epidural steroid injections in 72 patients with either spinal stenosis or acute herniated nucleus pulposus (HNP). Non-fluoroscopically guided interlaminar injections of either 1) methylprednisone, procaine and saline or 2) saline and procaine were performed. No statistically significant differences were found between the control and experimental patients in either the DLSS or HNP groups. Longterm follow-up, averaging 20 months, revealed 5 successes in the 23 stenosis patients who received epidural steroid injections ( 2 of 14 in the placebo group improved). Success was defined as a $75 \%$ improvement in pre-injection symptoms.

In a more recent randomized, controlled, single blind trial, Fukusaki et al (23) assessed the role of epidural steroid injections for treating symptoms of pseudoclaudication due to DLSS. Fifty-three patients were divided into three groups and given two consecutive interlaminar epidural steroid injections consisting of either 1) saline, 2) local anesthetic or 3) steroid plus local anesthetic. Follow-up at 1 week, 1 month and 3 months assessed improvement in walking distance following epidural steroid injections. Short term improvement was found at one week in both the local anesthetic and steroid/local anesthetic groups but none of the groups enjoyed sustained improvement in walking distance at one or three months.

Only two studies have reported specifically on the use of caudal epidural steroid injections for DLSS. Hoogmarten and Morelle (20) conducted a non-controlled, non-blinded study of caudal injections in 49 patients with DLSS and achieved a $48 \%$ success rate. Ciocon (21) et al treated 30 patients with DLSS manifesting as radicular symptoms. He reports in a descriptive, prospective, non-controlled trial, significant pain relief up to 10 months following injection. Unfortunately, the outcome measure used in the study, the overall change in a 5 point VNS score across the population is not a methodologically valid or intrinsically meaningful measure. Therefore very little can be gleaned from this study regarding the role of epidural steroid injections in DLSS management.

None of the above studies used fluoroscopic guidance for performing epidural steroid injections and thus their findings remain open to question since, as noted earlier, injectate is frequently misplaced in the absence of radiographic documentation. Only one study, by Botwin et al (22), specifically examined fluoroscop- 
ically guided epidural steroid injections for DLSS. In a prospective cohort study, 34 patients with unilateral radicular pain from DLSS underwent fluoroscopically guided transforaminal injections at the most symptomatic level. At one year follow-up, with an average of 1.9 injections per patient, $75 \%$ of patients continued to have $>50 \%$ pain reduction on the VNS scale compared to their pre-injection pain level. Additionally, $64 \%$ claimed improved walking tolerance, while $57 \%$ noted improved standing tolerance at one year.

To our knowledge, our retrospective study is the first to specifically examine the role of caudally placed epidural steroid injections performed under fluoroscopy in the management of DLSS. At an average follow-up time of 2.5 years, we found that $35 \%$ of patients reported at least a $50 \%$ improvement in pain, $36 \%$ noted a functional improvement of 2 points or more on the RMDQ and $42 \%$ were satisfied with the procedure per the NASS assessment.

Our outcomes are consistent with those found in a small retrospective analysis by Simotas et al (16). Although his evaluation of 49 patients with DLSS looked more generally at conservative management of the condition, the majority $(78 \%)$ did undergo fluoroscopic epidural steroid injections by either the transforaminal or caudal route. The average follow-up period of 33 months is nearly identical to that of our study (32 months). When one considers the relatively worse outcomes found in these two studies compared to those seen in Botwin et al (22) it is tempting to ascribe this difference to the relatively shorter follow-up time in Botwin's study. However, if this accounts for the difference then we should have noted a negative correlation between followup time and successful outcomes in our study. The difference also may be related to the total number of injections given. In our study, patients received an average of 1.6 injections. Botwin's study population underwent an average of 1.9 injections. It may be the case that we under-treated our patients. In fact, $58 \%$ of our patients underwent only one epidural steroid injection. Less than 12 percent received more than 2 injections. That we failed to find a positive correlation between the number of injections given and successful outcomes may reflect a lack of study power or a true lack of correlation.

Another explanation for Botwin et al (22) superior outcomes may be that transforaminal injections are a more efficacious injection route than the caudal route in the case of unilateral symptoms from DLSS. Such a possibility underscores the importance of conducting studies such as the present one. If we hope to achieve the best outcomes possible, precise evaluation of the best epidural steroid delivery routes for specific conditions is necessary. Ideally, studies of this kind should be performed in a prospective, randomized, controlled and blinded fashion.

The relatively higher success which we saw in our patients with spondylolisthesis seems counter-intuitive. Stenosis results from bony and soft tissue changes that lead to spinal canal narrowing. Obviously, spondylolisthesis represents a bony cause. As such it is less likely to resolve over time, whereas soft tissue changes such as disk bulges do tend to improve over time. If one keeps in mind the fact that degree of overall spinal canal narrowing does not correlate with symptom severity, then it becomes less difficult to understand how spondylolisthesis may respond to epidural steroid injections. It may be that mechanical compression with stenosis is not so much the direct source of pain as is the inflammatory reaction caused by the compression. The mechanical irritation of the dura and nerve root sheaths of the cauda equina may result in hyperemia, venous congestion, and leakage of biochemical and neurochemical inflammatory mediators, all of which contribute to painful nerve root edema (6, $25,22)$. It has also been demonstrated that herniated disk material is high in potent inflammatory and nociceptive mediators such as phospholipase A2 (37), leukotriene B4 and thromboxane B2 (38). One can speculate that the condition of spondylolisthesis places stress on the disk such that leakage of internal disk material occurs. The presumed effect of steroids in ameliorating stenotic symptoms may lie primarily in their role as anti-inflammatory agents. Corticosteroids inhibit inflammation by several mechanisms, including inhibition of leukocyte aggregation, prevention of granulocyte, mast cells, and macrophages degranulation, lysosomal membrane stabilization, and impairment of prostaglandin synthesis. Additionally, corticosteroids block nociceptive C-fiber transmission, resulting in direct pain pathway inhibition (39).
If spondylolisthesis activates the inflammatory cascade more so than other causes of stenosis, this could explain the greater rate of response to epidural steroid injections seen in this sub-population. In addition, response to steroids may also be a function of the extent of dural involvement in the inflammatory process. Stenosis caused by a slippage of one vertebral body on another results in a more focal involvement of the cauda equina caught between end plates than diffuse, multilevel stenosis. Unfortunately, no previous studies of spondylolisthesis and epidural steroid injections exist with which we may compare our findings.

A more mundane possibility is that the positive correlation between spondylolisthesis and successful outcomes is merely accidental. If so, however, the underlying responsible variable was not identified in our analysis. The spondylolisthetic population differed from the rest of the study population only in terms of age (average of 4 years older) and a greater frequency of single level verses multiple level stenosis. Neither of these variables correlated significantly with outcomes. Further studies would help to elucidate the role of caudal epidural steroid injections in specific etiologies of DLSS.

Besides spondylolisthesis, no other patient attribute distinguished responders from non-responders. Other than slightly younger age (63 verses 69 years), we found no significant differences between those patients who eventually underwent surgery and those who continued conservative management. The difference in ages between these groups may be the result of younger patients who have less co-morbidity and were preferentially selected as optimal surgical candidates.

Responses to epidural steroid injections were not significantly different when comparing patients with axial low back pain and those with radicular leg pain. Patients with DLSS resulting in axial pain of greater severity than leg pain have been found to respond more poorly to surgery (11). Additionally, a recent study by Southern et al (40) examined patients with predominantly axial low back pain of presumed discogenic origin (stenosis and spondylolisthesis were excluded) and found an overall poor response to fluoroscopic caudal epidural steroid injections. Our failure to corroborate a weaker response amongst stenosis patients with predominantly axial pain may be due to 
the efficacy of caudal epidural steroid injections in this subpopulation. Alternatively, it may have been due to a lack of power to detect a difference between small groups. This lack of power may have affected the sub-group analyses in general. This study suffers the limitations inherent to all retrospective analyses including selection bias, lack of blinding, lack of a control group, and limitations in power mentioned above.

\section{CONCLUSION}

Fluoroscopically guided, caudally placed epidural injections represent a relatively safe option for the management of DLSS generated pain. Many of our patients continued to enjoy improved function and diminished pain years after the epidural steroid injections were performed. The present study demonstrates that patients with spinal stenosis, particularly those with concomitant spondylolisthesis, are most likely to obtain benefit from caudal epidural steroid injections.

Author Affiliation:
Lisha Barré, MD
Physiatry Department
Hospital for Special Surgery
535 East $70^{\text {th }}$ St
New York, NY 10021
E-mail: lbarre@hotmail.com
Gregory E Lutz, MD
Physiatrist-in-chief
Hospital for Special Surgery
Associate Professor of Rehabilitation
Medicine
Weill Medical College of Cornell
University
550 East 70th Street
New York, NY 10021
E-mail: lutzg@hss.edu
Daniel Southern, MD
Physiatry Department
Hospital for Special Surgery
535 East 70
New Yt
Grant NY 10021
Physiatry Department
Hospital for Special Surgery
535 East 70
New York, NY 10021
E-mail: Coopergr1@yahoo.com

\section{References}

1. Botwin KP, Gruber RD. Lumbar spinal stenosis: anatomy and pathogenesis. Phys Med Rehabil Clin N Am 2003; 14:11-15.

2. Fritz JM, Delitto A, Welch WC et al. Lumbar spinal stenosis: A review of current concepts in evaluation, management, and outcome measurements. Arch Phys Med Rehabil 1998; 79: 700-708.

3. Kent DL, Haynor DR, Larson EB et al. Diagnosis of lumbar spinal stenosis in adults: A meta-analysis of the accuracy of CT, MRI, and myelography. Am / Roentgenol 1992; 158:1135-1144.

4. Amundson T, Weber H, Lilleas F, et al. Lumbar spinal stenosis: clinical and radiologic features. Spine 1995; 20:1178-1186.

5. Boden S, Davis D, Dina T et al. Abnormal magnetic-resonance scans of the lumbar spine in asymptomatic subjects. J Bone Joint Surg 1999; 72A:403-408.

6. Simotas AC. Nonoperative treatment for lumbar spinal stenosis. Clin Orthop 2001; 384:153-161.

7. Deyo RA, Cherkin DC, Loeser JD et al. Morbidity and mortality in association with operations in the lumbar spine. J Bone Joint Surg 1992; 74A:536-543.

8. Turner JA, Ersek M, Herron L et al. Surgery for lumbar spinal stenosis: attempted meta-analysis of the literature. Spine 1992; 17:1-8.

9. Sheehan J, Shaffrey C, Jane J. Degenerative lumbar stenosis: The neurosurgical perspective. Clin Orthop 2001; 384:61-74.

10. Katz J, Lipson $S$, Larson $M$ et al. The outcome of decompressive laminectomy for lumbar stenosis. J Bone Joint Surg 1996; 73A:809-816.

11. Atlas SJ, Robert KB, Robson D et al. Surgical and non-surgical management of lumbar spinal stenosis: Four year outcomes from the Maine lumbar spine study. Spine 2000; 25:556-562.

12. Zak PJ. Surgical management of spinal stenosis. Phys Med Rehabil Clin N Am 2003; 14:143-155.

13. Johnsson KE. Rosen I, Uden A. The natural course of lumbar spinal stenosis. Clin Orthop 1992; 279:82-86.

14. Atlas SJ, Deyo RA, Keller RB et al. The Main lumbar spine study part III: 1-year outcomes of surgical and non-surgical management of lumbar spinal stenosis. Spine 1996; 21:1787-1794.

15. Onel D, Sari H, Donmez C. Lumbar spinal stenosis: Clinical/radiographic therapeutic evaluation in 145 patients. Conservative treatment or surgical intervention? Spine 1993; 18:291-298.

16. Simotas AC, Dorey FJ, Hansraj K et al. Nonoperative treatment for lumbar spinal stenosis: Clinical outcome results and a 3 year survivorship analysis. Spine 2000; 25:197-204.

17. Swezey RL. Outcomes for lumbar stenosis: A five-year follow-up study. I Clin Rheumatol 1996; 2:129-134.
18. Amundson $\mathrm{T}$, Weber $\mathrm{H}$, Nordal $\mathrm{HJ}$ et al. Lumbar spinal stenosis: Conservative or surgical management? A prospective 10year study. Spine 2000; 25:1424-1436.

19. Whitman JM, Flynn TW, Fritz JM. Non-surgical management of patients with lumbar spinal stenosis: a literature review and a case series of three patients managed with physical therapy. Phys Med Rehabil Clin N Am 2003; 14: 77-101.

20. Hoogmartens M, Morelle P. Epidural injection in the treatment of spinal stenosis. Acta Orthop Belg 1987; 53: 409-411.

21. Ciocon JO, Galindo-Ciocon D, Amaranth $L$ et al. Caudal epidural blocks for elderly patients with lumbar canal stenosis. I Am Geriatr Soc 1994; 42:593-596.

22. Botwin KP, Gruber RD, Bouchlas CG et al. Fluoroscopically guided lumbar transforaminal epidural steroid injections in degenerative lumbar stenosis: An outcome study. Am J Phys Med Rehabil 2002; 81: 898-905.

23. Fukusaki M, Kobayashi I, Hara T et al. Symptoms of spinal stenosis do not improve after epidural steroid injection. Clin J Pain 1998; 14:148-151.

24. Cuckler JM, Bernin PA, Wisel SW et al. The use of epidural steroids in the treatment of lumbar radicular pain. J Bone Joint Surg Am 1985; 67: 63-66.

25. Botwin KP, Gruber RD. Lumbar epidural steroid injections in the patient with lumbar spinal stenosis. Phys Med Rehabil Clin N Am 2003; 14:121-141.

26. Bogduk N, Brazenor G, Christophides N et al. Epidural steroids in the management of low back pain and sciatica of spinal origin. Report of the working party. Sydney: $\mathrm{Na}$ tional Health and Medical Research Council 1993:102-106.

27. Lutz GE, Vad VB, Wisneski RJ. Fluoroscopic transforaminal lumbar epidural steroids: An outcome study. Arch Phys Med Rehabil 1998; 79:1362-1366.

28. Singh V, Manchikanti L. Role of caudal epidural injections in the management of chronic low back pain. Pain Physician 2002; 5:133-148.

29. El-Khoury G, Ehara S, Weinstein JN et al. Epidural steroid injection: A procedure ideally performed with fluoroscopic control. Radiology 1988; 168:554-557.

30. Renfrew DL, Moore TE, Kathol MH et al. Correct placement of epidural steroid injections: Fluoroscopic guidance and contrast administration. Am / Neuroradiol 1991; 12:1003-1007.

31. Mehta M, Salmon N. Extradural block: Confirmation of the injection site by $\mathrm{x}$-ray monitoring. Anesthesia 1985; 40:10091012.

32. Stitz MY, Hillel SM. Accuracy of blind versus fluoroscopically guided caudal epidural injection. Spine 1999; 24:1371-1376.

33. White AH, Derby R, Wynne G. Epidural injections in the diagnosis and treatment of low back pain. Spine 1980; 5:78-86. 
34. Robecchi A, Capra R. Lidrocortisone (Composto F), rime esperinze cliniche in campo reumatologico. Minerva Med 1952; 98: 1259-1263.

35. Benson H. Epidural steroid injections for low back pain and lumbosacral radiculopathy. Pain 1986; 24: 244-295.

36. Woodward JL, Herring SA, Windsor RE. Epidural Procedures in Spine Pain Management. In Lennard TA, ed. Pain Proce- dures in Clinical Practice, 2nd ed. Philadelphia, Hanley and Belfus, Inc., 2000, pp 341-376.

37. Saal JS, Franson RC, Dobrow R et al. High levels of inflammatory phospholipase A2 activity in lumbar disk herniations. Spine 1990; 15:674-678.

38. Nygard OP, Mellgren SI, Osterud B. The inflammatory properties of contained and noncontained lumbar disc herniation.
Spine 1997; 22: 2484-2488.

39. Cannon DT, Aprill CN. Lumbosacral epidural steroid injections. Arch Phys Med Rehabil 2000; 81: 87-98.

40. Southern D, Lutz GE, Cooper G et al. Are fluoroscopic caudal epidural steroid injections effective for managing chronic low back pain? Pain Physician 2003; 6:167-172. 
